\title{
DETERMINE VARIATION OF POISSON RATIOS AND THERMAL CREEP STRESSES AND STRAIN RATES IN AN ISOTROPIC DISC
}

\author{
Nishi Gupta1, Satya Bir Singh ${ }^{2}$, Pankaj Thakur ${ }^{3 *}$ \\ ${ }^{1,2}$ Department of Mathematics, Punjabi University Patiala, Punjab 147002, India \\ ${ }^{3}$ Department of Mathematics, IEC University, Baddi, Solan, Himachal Pradesh 174103, India \\ *Corresponding author; E-mail: pankaj_thakur15@yahoo.co.in
}

(Received April 5, 2016)

\begin{abstract}
Seth's transition theory is applied to the problem of thermal creep transition stresses and strain rates in a thin rotating disc with shaft having variable density by finite deformation. Neither the yield criterion nor the associated flow rule is assumed here. The results obtained here are applicable to compressible materials. If the additional condition of incompressibility is imposed, then the expression for stresses corresponds to those arising from Tresca yield condition. Thermal effect decreased value of radial stress at the internal surface of the rotating isotropic disc made of compressible material as well as incompressible material and this value of radial stress further much increases with the increase in angular speed. With the introduction of thermal effects, the maximum value of strain rates further increases at the internal surface for compressible materials as compare to incompressible material.
\end{abstract}

Keywords: strain rates, displacement, angular speed, disc, thermal stresses.

\section{INTRODUCTION}

Rotating discs provide an area of research and study due to their vast utilization in rotating machinery such as compressor, turbo generators, pumps, compressors, flywheels, shrink fits, automotive braking systems, ship propellers, computer disc drives, steam and gas turbine rotors. Theoretical investigation of the stresses and strain rates in annular discs rotating at high speeds have received widespread attention due to a large number of applications in mechanical and structural engineering. They are usually operated at relatively higher angular speed and high temperature. Therefore the prediction of long term steady state creep deformation is very important for these applications.

The classical theories of creep start with the assumptions of constitutive equations for creep and the classical theories of plasticity need an extra relation called the yield condition in addition to the flow rules. The description of the deformations in a solid subjected to external forces is thus given by a different set of equations for elastic, plastic and creep deformations. Solutions for thin isotropic discs can be found in most of the standard creep text books (Lubhan, 1961; Oduist, 1974; Karaus, 1980; Boyle, 1983; NABARro, 1995; PenNy, 1995; HOFFMAN, 2012). In most of engineering application, the disc has to operate under elevated temperature and is simultaneously subjected to high stresses caused by disc rotation at high 
speed (LASKAJ, 1999). As a result of severe mechanical and thermal loading, the material of disc undergoes creep deformation, thereby affecting performance of the system (FARHI et al. 2004, 2008). In recent years, the problem of creep in rotating discs made of functionally graded materials, subjected to severe mechanical and thermal load, has attracted the interest to many researchers.

GUPTA et al. (2007) study network modeling of creep behavior in rotating composite disc. DEEPAK et al. (2010) investigated the problem creep modeling in functionally graded rotating disc of variable thickness. DEEPAK et al. (2015) discuss the problem creep behavior of rotating FGM disc with linear and hyperbolic thickness profiles. SETH (1962) investigated Transition theory of elastic-plastic deformation, creep and relaxation. GUPTA et al. (1979, 1981, 2000, 2007, 2008) analyzed creep transition in thin rotating disc and cylinder having various conditions. SHKULA (1996) investigated creep transition in a thin rotating nonhomogeneous disc by using Seth theory. THAKUR (2010) analyzed creep transition stresses in a thin rotating disc with shaft by finite deformation under steady state temperature. SHARMA Sanjeev et al., (2010, 2013) analyzed creep deformation in a thin rotating disk of exponentially varying thickness with inclusion and edge load by using Seth transition theory. THAKUR et. al. (2015) study thermal creep stresses and strain rates in a circular disc with shaft having variable density by using Seth transition theory. WAHL (1956) has investigated creep deformation in rotating discs assuming small deformation, incompressibility condition, Tresc'a yield criterion, its associated flow rule and a power strain law.

The necessity of increasing use of ad-hoc semi-empirical laws in the classical theory of elastic-plastic and creep transition lies in the fact that the latter does not recognize the existence of the transition state between elastic and plastic ones and then creep. We have shown in this research paper that assumptions of yield conditions in such problems become unnecessary once we recognize that the transition from plastic state to creep, as explained by Seth, is an asymptotic process and that transition state is a separate state which cannot be replaced by a yield surface as has always been done in the current literature. This treatment in the classical theory amounts to divide two extreme properties of a material by a sharp line which is physically impossible. It has been clear from our work that identification of the transition state is basically important.

There are, at present, three ways to identify the transition state. The most general one among all is the vanishing of the Jacobian of transformation from elastic state to plastic state and plastic to creep. An invariant relation among the strain (stress) invariants is obtained from this condition and it is found that most of the yields conditions present in current literature are obtainable from it as special cases.

Also our results include the Bauschinger's effect while the classical yield conditions fail to account for it. The classical theory of elasticity, plasticity and creep makes use of linear strain measure. But we have shown that transition fields are sub-harmonic (super-harmonic) fields and that they are non-linear and non-conservative in character and hence it is very important that a non-linear strain measure such as the Almansi measure should be used in the constitutive equation. The recognition of transition state or mid-zone as a separate state necessitates showing the existence of the constitutive equation for that state. In this context, we have used Seth's transition theory to obtain the stresses and strains in the transition state and the same may be obtained for the plastic state when a certain parameter $c(1-c / 2-v)$; where $v$ is the Poisson's ratio of the material, is made to approach zero. From these solutions the constitutive equations for both transition and plastic states are obtained, the latter takes the form of the Levy-von-Mises equation. In nature transitions do occur frequently and the existing classical theory fails to explain them successfully. Thus the transition theory, as it stands, now can be fruitfully exploited to explain a variety of physical phenomena and hence 
has a very wide application in all applied sciences. Seth's transition theory does not acquire any assumptions like an yield condition, incompressibility condition and thus poses and solves a more general problem from which cases pertaining to the above assumptions can be worked out. This theory utilizes the concept of generalized strain measure and asymptotic solution at critical points or turning points of the differential equations defining the deformed field and has been successfully applied to a large number of problems (SETH, 1962, 1966, 1970, 1972, 1974; GuPTA et al., 1979, 1981, 2000, 2007, 2008; ShKULA, 1996; DEEPAK et. al., 2010, 2015; SHARMA et al., 2010, 2013; THAKUR, 2010, 2015). In this paper we discuss numerical study of possion ratios and thermal stress and strain rates in an isotropic disc by using Seth transition theory.

\section{GOVERNING EQUATIONS OF THE PROBLEM}

Consider a circular disc with central bore of radius $a$ and external radius $b$ and having uniform thickness rotating with an angular velocity $\omega$ of gradually increasing magnitude about an axis perpendicular to its plane and passing through the center. The thickness of the disc is assumed sufficiently small so that it is effectively in a state of plane stress $\left(T_{z z}\right.$ $=0$ ). The temperature at the central bore of the disc is $\Theta_{0}$ as shown in Figure 1.

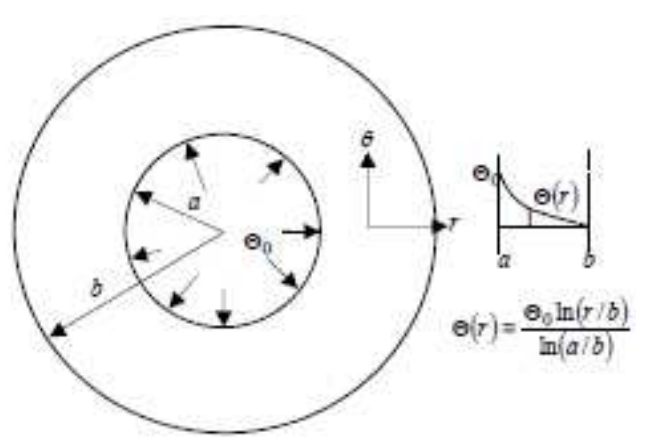

Figure 1. Geometry of disk

Displacement Coordinate: The displacement components in cylindrical polar co-ordinate are given by (SETH, 1962, 1966, 1970, 1972):

$u=r(1-\beta), v=0, w=d z$

where $u, v, w$ (displacement components); $\beta$ is position function, depending on $r=$ $\sqrt{x^{2}+y^{2}}$ only, and $d$ is a constant. The generalized components of strain are given by Seth's (1972):

$e_{r r}=\frac{1}{n}\left[1-\left(r \beta^{\prime}+\beta\right)^{n}\right], e_{\theta \theta}=\frac{1}{n}\left[1-\beta^{n}\right], e_{z z}=\frac{1}{n}\left[1-(1-d)^{n}\right], e_{r \theta}=e_{\theta z}=e_{z r}=0$

where $r, \theta, z$ be polar co-ordinates and $\beta^{\prime}=d \beta / d r$.

Stress-Strain Relation: The stress -strain relations for thermo elastic isotropic material are (Parkus, 1976):

$T_{i j}=\lambda \delta_{i j} I_{1}+2 \mu e_{i j}-\xi \Theta \delta_{i j},(i, j=1,2,3)$

where $T_{i j}$ are the stress components, $\lambda$ and $\mu$ are Lame's constants, $I_{1}=e_{k k}$ is the first strain invariant, $\delta_{i j}$ is the Kronecker's delta, $\xi=\alpha(3 \lambda+2 \mu), \alpha$ being the coefficient of thermal expansion, and $\Theta$ is the temperature. Further, $\Theta$ has to satisfy: $\nabla^{2} \Theta=0 \Rightarrow$ $\frac{d^{2} \Theta}{d r^{2}}+\frac{1}{r} \frac{d \Theta}{d r} \equiv \frac{1}{r} \frac{d}{d r}\left(r \frac{d \Theta}{d r}\right)=0$ or $\frac{d \Theta}{d r}=\frac{k_{1}}{r} ;$ which has solutions: $\Theta=k_{1}\left(\log r+k_{2}\right)$ 
where $k_{1}$ and $k_{2}$ are constant of integration and can determined from the boundary condition. Substituting (Equation (2)) in (Equation (3)), the stresses are obtained as:

$$
\begin{aligned}
& T_{r r}=\frac{2 \mu}{n}\left[3-2 c-\beta^{n}\left\{1-c+(2-c)(P+1)^{n}+\frac{n c \xi \Theta}{2 \mu \beta^{n}}\right\}\right], \\
& T_{\theta \theta}=\frac{2 \mu}{n}\left[3-2 c-\beta^{n}\left\{2-c+(1-c)(P+1)^{n}+\frac{n c \xi \Theta}{2 \mu \beta^{n}}\right\}\right], T_{r \theta}=T_{\theta z}=T_{z r}=T_{z z}=0
\end{aligned}
$$

where $c$ is the compressibility factor of the material in term of Lame's constant, and are given by $c=2 \mu / \lambda+2 \mu$.

Equation of equilibrium: The radial equilibrium of an element of the rotating disc requires:

$\frac{d}{d r}\left(r T_{r r}\right)-T_{\theta \theta}+\rho \omega^{2} r^{2}=0$

where $T_{r r}$ and $T_{\theta \theta}$ are the radial and circumferential stresses, $\rho$ is the material density of the disk and $\omega$ is the constant angular velocity.

Boundary conditions: The temperature satisfying Laplace (Equation (4)) with boundary condition:

$\Theta=\Theta_{0}$ and $u=0$ at $r=a, \Theta=0$ and $T_{r r}=0$ at $r=b$,

where $\Theta_{0}$ is constant, given by (Parkus, 1976): $k_{1}=\frac{\Theta}{\log (a / b)}$ and $k_{2}=\log b$. Substituting $k_{1}$ and $k_{2}$ from (Equation (4)), we get: $\Theta=\frac{\Theta_{0} \log (r / b)}{\log (a / b)}$

Critical points or Turning points: Using (Equations (5) \& (8)) in (Equation (6)), we get a non- linear differential equation in $\beta$ as:

$$
(2-c) n \beta^{n+1} P(P+1)^{n-1} \frac{d P}{d \beta}=\frac{n \rho \omega^{2} r^{2}}{2 \mu}-\frac{n c \xi \bar{\Theta}_{0}}{2 \mu}+\beta^{n}\left\{1-(P+1)^{n}-n P\left[1-c+(2-c)(P+1)^{n}\right]\right\}
$$

where $\bar{\Theta}_{0}=\Theta_{0} / \log (a / b)$ and $r \beta^{\prime}=\beta P(P$ is function of $\beta$ and $\beta$ is function of $r)$.

Transition points of $\beta$ in (Equation (9)) are $P \rightarrow-1$ and $P \rightarrow \pm \infty$.

\section{SOLUTION OF THE PROBLEM}

For finding the thermal creep stresses and strain rates, the transition function is taken through principal stress difference (see SETH, 1962, 1966, 1970, 1972, 1974; GUPTA et al. 1979, 1981, 2000, 2007, 2008; SHKULA, 1996; SANJEEV et al., 2010, 2013; DEEPAK et. al. 2010, 2015; THAKUR, 2010, 2015, 2016) at the transition point $P \rightarrow-1$ we define the transition function $\square$ as:

$\square=T_{r r}-T_{\theta \theta}=\frac{2 \mu \beta^{n}}{n}\left[1-(P+1)^{n}\right]$

where $\square$ is function of $r$ only and $\square$ is dimension. 
Taking the logarithmic differentiating of eq. (10) with respect to $r$ and substituting eq. (9) and taking asymptotic value $P \rightarrow-1$, we get:

$$
\frac{d}{d r}(\ln \square)=-\frac{1}{r(2-c)}\left\{n(3-2 c)+1+\frac{n \rho w^{2} r^{2+n}}{2 \mu D^{n}}-\frac{n c \xi \bar{\Theta}_{0}}{2 \mu \beta^{n}(2-c)}\right\}
$$

Asymptotic value of $\beta$ as $P \rightarrow-1$ is $D / r ; D$ being a constant. Integrating equation (Equation (11)) with respect to $r$ and using(Equation (10)), we get

$$
\square=T_{r r}-T_{\theta \theta}=A r^{k_{3}} \exp \left(\phi r^{n+2}+\psi r^{n}\right),
$$

where $A, f$ and $g$ are constant of integrations, which can be determine by boundary condition and

$v=\frac{1-c}{2-c}$ be Poisson ratio's, $c \xi=\alpha E /(1-v)$,

$k_{3}=-[(n+1)+v(n-1)], \psi=\frac{\alpha \bar{\Theta}_{0}(1+v)}{D^{n}}, \phi=-\frac{n \omega^{2} \rho(1-v)}{2 \mu D^{n}(n+2)}=-\frac{n \omega^{2} \rho\left(1-v^{2}\right)}{E D^{n}(n+2)}$. From

(Equations (10) and (12)), we have

$T_{r r}-T_{\theta \theta}=A r^{k_{3}} \exp \left(\phi r^{n+2}+\psi r^{n}\right)$

Substituting (Equation (13)) in (Equation (6)), we get:

$$
T_{r r}=B-A \int r^{k_{3}-1} \exp \left(\phi r^{n+2}+\psi r^{n}\right) d r-\frac{\rho \omega^{2} r^{2}}{2}
$$

where $B$ is a constant of integration, which can be determine by boundary condition. Using boundary condition (Equation (7)) in (Equation (14)), we get $B=A \int_{r=b} r^{k_{3}-1} \exp \left(\xi r^{n+2}+\psi r^{n}\right) d r+\frac{\rho \omega^{2} r^{2}}{2}$.

Substituting the value of $B$ in (Equation (14)), we get:

$$
T_{r r}=-A \int_{r}^{b} r^{k_{3}-1} \exp \left(\xi r^{n+2}+\psi r^{n}\right) d r+\frac{\rho \omega^{2}\left(b^{2}-r^{2}\right)}{2}
$$

Substituting (Equation (15) in (Equation (13)), we get:

$$
T_{\theta \theta}=-A\left(\int_{r}^{b} r^{k_{3}-1} \exp \left(\xi r^{n+2}+\psi r^{n}\right) d r-r^{k_{3}} \exp \left(\xi r^{n+2}+\psi r^{n}\right)\right)+\frac{\rho \omega^{2}\left(b^{2}-r^{2}\right)}{2}
$$

Comparing (Equations (10) and (13)) and then taking asymptotic value $P \rightarrow-1$, we get:

$$
\beta=\left[\frac{n(1+v)}{E} A^{k_{3}-1} \exp \left(\xi r^{n+2}+\psi r^{n}\right)\right]^{1 / n}
$$

where $2 \mu=E /(1+v)$ is the Young's modulus in term of Poisson's ratio. Using (Equation (17)) in (Equation (1)), we get

$$
u=r-r\left[\frac{n(1+v)}{E} A r^{k_{3}} \exp \left(\xi r^{n+2}+\psi r^{n}\right)\right]^{1 / n}
$$

where $u$ be the displacement component. 
Using boundary condition (Equation (7)) in (Equation (18)), we get; $A=\frac{E}{n(1+v) a^{k_{3}} \exp \left(\phi a^{n+2}+\xi a^{n}\right)}$. Substituting the value of constant $A$ in (Equations (15), (16) and (18)), we get:

$$
\begin{aligned}
& T_{\theta \theta}=\left\{\frac{E}{n(1+v) a^{k_{3}} \exp \left(\phi a^{n+2}+\psi a^{n}\right)}\left[\int_{r}^{b}-r^{k_{3}} \exp \left(\phi r^{n+2}+\psi r^{n}\right)\right]+\frac{\rho \omega^{2}\left(b^{2}-r^{2}\right)}{2}\right\} \\
& T_{r r}=\frac{E}{n(1+v) a^{k_{3}} \exp \left(\phi a^{n+2}+\psi a^{n}\right)} \int_{r}^{b}\left[r^{k_{3}-1} \exp \left(\phi r^{n+2}+\psi r^{n}\right)\right] d r+\frac{\rho \omega^{2}\left(b^{2}-r^{2}\right)}{2} \\
& u=r-r\left[\frac{r^{k_{3}} \exp \left(\xi r^{n+2}+\psi r^{n}\right)}{a^{k_{3}} \exp \left(\xi a^{n+2}+\psi a^{n}\right)}\right]^{\frac{1}{n}} .
\end{aligned}
$$

(Equations. (19) - (21)) give creep stresses and displacement for a thin rotating disc with shaft at temperature $\Theta_{0}$. We introduce the following non-dimensional components as: $R=r / b, R_{0}=a / b, \sigma_{r}=T_{r r} / E, \sigma_{\theta}=T_{\theta \theta} / E, \Omega^{2}=\rho \omega^{2} b^{2} / E, \quad \bar{u}=u / b \quad$ and $\quad \alpha \Theta_{0}=\Theta_{1}$. (Equations (19) - (21)) in non-dimensional form become:

$$
\begin{aligned}
& \sigma_{\theta}=\frac{1}{n(1+v) R_{0}^{k_{3}} \exp \left(\xi_{1} R_{0}^{n+2}+\psi_{1} R_{0}^{n}\right)} \int_{R}^{1}\left[\begin{array}{c}
R^{k_{3}-1} \exp \left(\xi_{1} R^{n+2}+\psi_{1} R^{n}\right) \\
-R^{k_{3}} \exp \left(\xi_{1} R^{n+2}+\psi_{1} R^{n}\right)
\end{array}\right] d R+\frac{\Omega^{2}\left(1-R^{2}\right)}{2} \\
& \sigma_{r}=\frac{1}{n(1+v) R_{0}^{k_{3}} \exp \left(\xi_{1} R_{0}^{n+2}+\psi_{1} R_{0}^{n}\right)} \int_{R}^{1} R^{k_{3}-1} \exp \left(\xi_{1} R^{n+2}+\psi_{1} R^{n}\right) d R+\frac{\Omega^{2}\left(1-R^{2}\right)}{2} \\
& \bar{u}=R-R\left[\frac{R^{k_{3}} \exp \left(\xi_{1} R^{n+2}+\xi_{1} R^{n}\right)}{R_{0}^{k_{3}} \exp \left(\xi_{1} R_{0}^{n+2}+\psi_{1} R_{0}^{n}\right)}\right]^{\frac{1}{n}}
\end{aligned}
$$

where $\xi_{1}=-\frac{n \Omega^{2}\left(1-v^{2}\right) b^{n}}{D^{n}(n+2)} ; \psi_{1}=\frac{\Theta_{1}(1+v) b^{n}}{D^{n} \ln R_{0}} \quad$ (Constants); $\sigma_{\theta} \quad$ (Tangential stresses); $\sigma_{r}$ (Radial stress); $R=r / b$ and $R_{0}=a / b$ (Radii ratios).

Fully-Plastic state: For a disc made of incompressible material $(v \rightarrow 1 / 2$ or $c=0)$ (Equations (22) to (24)) become:

$$
\begin{aligned}
& \sigma_{\theta}=\frac{2}{3 n R_{0}^{k_{4}} \exp \left(\xi_{2} R_{0}^{n+2}+\psi_{2} R_{0}^{n}\right)} \int_{R}^{1}\left[\begin{array}{l}
R^{k_{4}-1} \exp \left(\xi_{2} R^{n+2}+\psi_{2} R^{n}\right) \\
-R^{k_{4}} \exp \left(\xi_{2} R^{n+2}+\psi_{2} R^{n}\right)
\end{array}\right] d R+\frac{\Omega^{2}\left(1-R^{2}\right)}{2} \\
& \sigma_{r}=\frac{2}{3 n R_{0}^{k_{4}} \exp \left(\xi_{2} R_{0}^{n+2}+\psi_{2} R_{0}^{n}\right)} \int_{R}^{1} R^{k_{4}-1} \exp \left(\xi_{2} R^{n+2}+\psi_{2} R^{n}\right) d R+\frac{\Omega^{2}\left(1-R^{2}\right)}{2} \\
& \bar{u}=R-R\left[\frac{R^{k_{4}} \exp \left(\xi_{2} R^{n+2}+\psi_{2} R^{n}\right)}{R_{0}^{k_{4}} \exp \left(\xi_{2} R_{0}^{n+2}+\psi_{2} R_{0}^{n}\right)}\right]^{\frac{1}{n}}
\end{aligned}
$$


where $\xi_{2}=-\frac{3 n \Omega^{2} b^{n}}{4 D^{n}(n+2)} ; k_{4}=-\frac{3 n+1}{2}$ and $\psi_{2}=\frac{\Theta_{1} b^{n}}{2 D^{n} \ln R_{0}}$.

\section{DISTRIBUTION VARIATION PARAMETER IN POISSON RATIOS:}

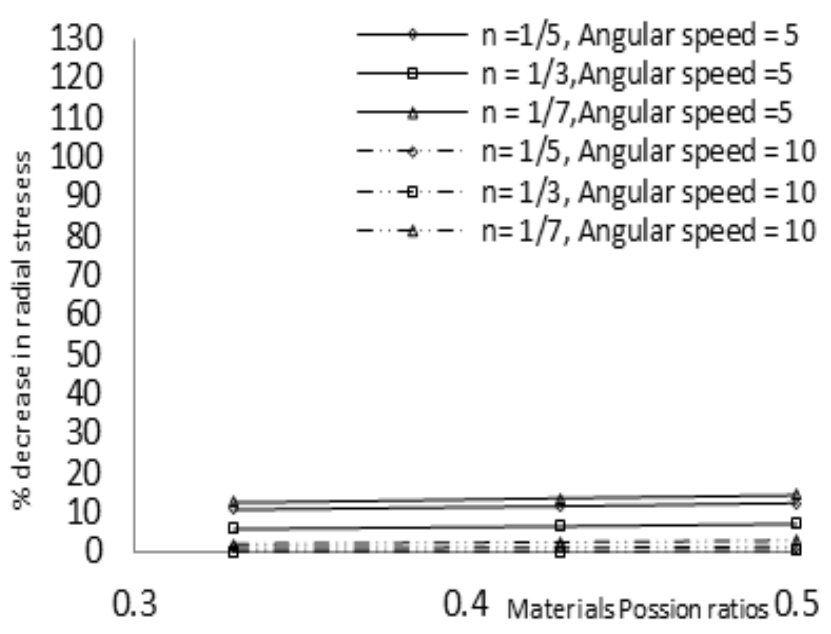

Figure 3(a). Percentage decrease in radial.

Versus materials Poisson ratios.

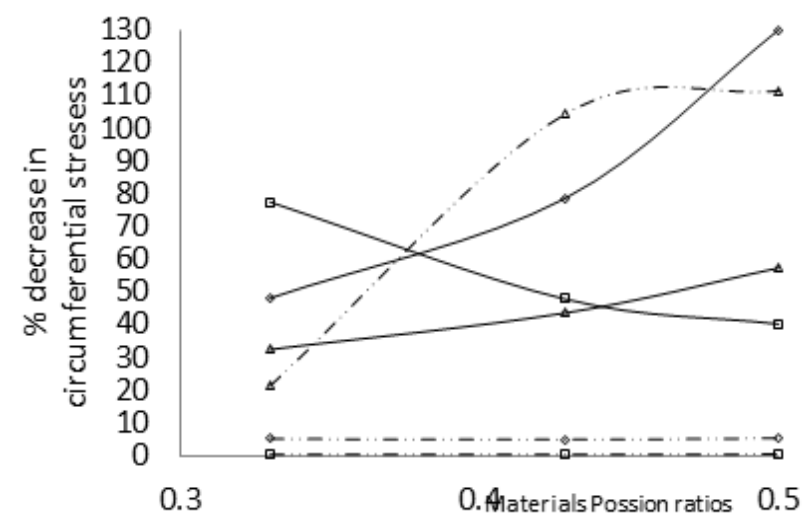

Figure 3(b). Percentage decrease in Circumferential stresses.

Versus materials Poisson ratios.

\section{ESTIMATION OF CREEP PARAMETERS}

When the creep sets in, the strains should be replaced by strain rates and the stress-strain relations (Equation (3)) become:

$\dot{e}_{i j}=\frac{1+v}{E} \sigma_{i j}-\frac{v}{E} \delta_{i j} T+\alpha \Theta$

where $\dot{e}_{i j}$ is the strain rate tensor with respect to flow parameter $t$. Differentiating (Equation (4)) with respect to time $t$, we get:

$\dot{e}_{\theta \theta}=-\beta^{n-1} \dot{\beta}$

For SWAINGER measure (i.e. $n=1$ ), (equation (29)) become : $\dot{\varepsilon}_{\theta \theta}=\dot{\beta}$. 
where $\dot{\varepsilon}_{\theta \theta}$ is the SWAINGER strain measure. From (Equation (10)) the transition value $\beta$ is given by:

$\beta=(n / 2 \mu)^{1 / n}\left[\sigma_{r r}-\sigma_{\theta \theta}\right]^{1 / n}$

Using (Equations (29)-(31)) in (Equation (28)), we get:

$$
\begin{aligned}
& \dot{\varepsilon}_{r r}=\left[n\left(\sigma_{r}-\sigma_{\theta}\right)(1+v)\right]^{\frac{1}{n}-1}\left[\sigma_{r}-v \sigma_{\theta}+\alpha \Theta\right] \\
& \dot{\varepsilon}_{\theta \theta}=\left[n\left(\sigma_{r}-\sigma_{\theta}\right)(1+v)\right]^{\frac{1}{n}-1}\left[\sigma_{\theta}-v \sigma_{r}+\alpha \Theta\right] \\
& \dot{\varepsilon}_{z z}=-\left[n\left(\sigma_{r}-\sigma_{\theta}\right)(1+v)\right]^{-1}\left[v\left(\sigma_{r}+\sigma_{\theta}\right)+\alpha \Theta\right]
\end{aligned}
$$

where $\dot{\varepsilon}_{r r} \dot{\varepsilon}_{\theta \vartheta}$ and $\dot{\varepsilon}_{z z}$ are strain rates tensor.

These are the constitutive equations used by ODQUIST (1974) for finding the creep stresses and strain rates provided we put $n=1 / N$.

\section{NUMERICAL RESULTS AND DISCUSSION}

For calculating stresses, strain-rates and displacement based on the above analysis, the following values have been taken $\Omega^{2}=\rho \omega^{2} b^{2} / E=5,10 v=0.5$ (incompressible material), $v=0.42857$ and 0.333 (compressible materials), $n=1 / 3,1 / 5,1 / 7$ (i.e $N=3,5,7$ ), $\alpha=5.0 \times 10^{-5} \mathrm{deg} F^{-1}$ (for Methyl Methacrylate; LeVITSKY et. al., 1975 ), $\Theta_{0}=0$ and $1,00,000^{0} F, \Theta_{1}=\alpha \Theta_{0}=0.00$ and 5 and $D=1$.

In classical theory measure $N$ is equal to $1 / n$. Definite integrals in the equations (22) and (23) have been solved by using Simpson's rule.

It has been seen from Fig. 4 and 5, curve have been between radial stresses versus temperature $\Theta_{1}=0,5$ for measure $n=1 / 7,1 / 5,1 / 3, \Omega^{2}=5,10$. With the thermal effect stresses must be decreases.

- For measure $n=1 / 7$, decrease percentage change are $-12.4 \%,-13.5 \%,-14.3 \%$ at angular speed $\Omega^{2}=5$ and $-1.9 \%,-2.4 \%,-2.8 \%$ (-ve sign indicates decease value) at angular speed $\Omega^{2}=10$ having possion ratios $v=0.33,0.428,0.5$.

- For measure $n=1 / 5$, decrease percentage change are $-10.6 \%,-11.5 \%,-12.3 \%$ at angular speed $\Omega^{2}=5$ and $-0.8 \%,-1 \%,-1.3 \%$ (-ve sign indicates decease value) at angular speed $\Omega^{2}=10$ as shown having possion ratios $v=0.33,0.428,0.5$.

- For measure $n=1 / 3$, decrease in percentage change are $-5.7 \%,-6.4 \%,-7 \%$ at angular speed $\Omega^{2}=5$ and $-0.1 \%,-0.12 \%,-0.2 \%$ (-ve sign indicates decease value) at angular speed $\Omega^{2}=10$ having possion ratios $v=0.33,0.428,0.5$.

From Table 1, it has been seen that thermal effect decreases the value of radial stresses as well as circumferential stresses at the internal surface for compressible material as compare to incompressible material for measure $n=1 / 7,1 / 5$ and 1/3. Percentage change in radial and circumferential stresses should be decreased with effect of temperature.

Curves are produced between stresses and displacement along the radii ratio $R=r / b$ (see Figures 5(a) and 5(b)) for rotating disc made of compressible as well as incompressible materials with angular speed $\Omega^{2}=5$ and 10. It is also observed from (Figures 5(a)-5(b)) that the radial stress has maximum value at the internal surface of the rotating disc made of 
compressible material (i.e. $v=0.33$ say Copper; 0.428 say saturated clay) as compare to incompressible materials (i.e. $v=0.5$ say rubber) for measure $n=1 / 7$ (i.e. $N=7$ ) at angular speed $\left(\Omega^{2}=5\right)$. The values of radial stress further increases at the internal surface with increase value of angular speed $\left(\Omega^{2}=10\right)$ for measure $n=1 / 7$ (i.e. $N=7$ ), $n=1 / 5$ (i.e. $N=5$ ) and $n=1 / 3$ (i.e. $N=3$ ) respectively. Thermal effect decreases the values of radial stress at the internal surface.

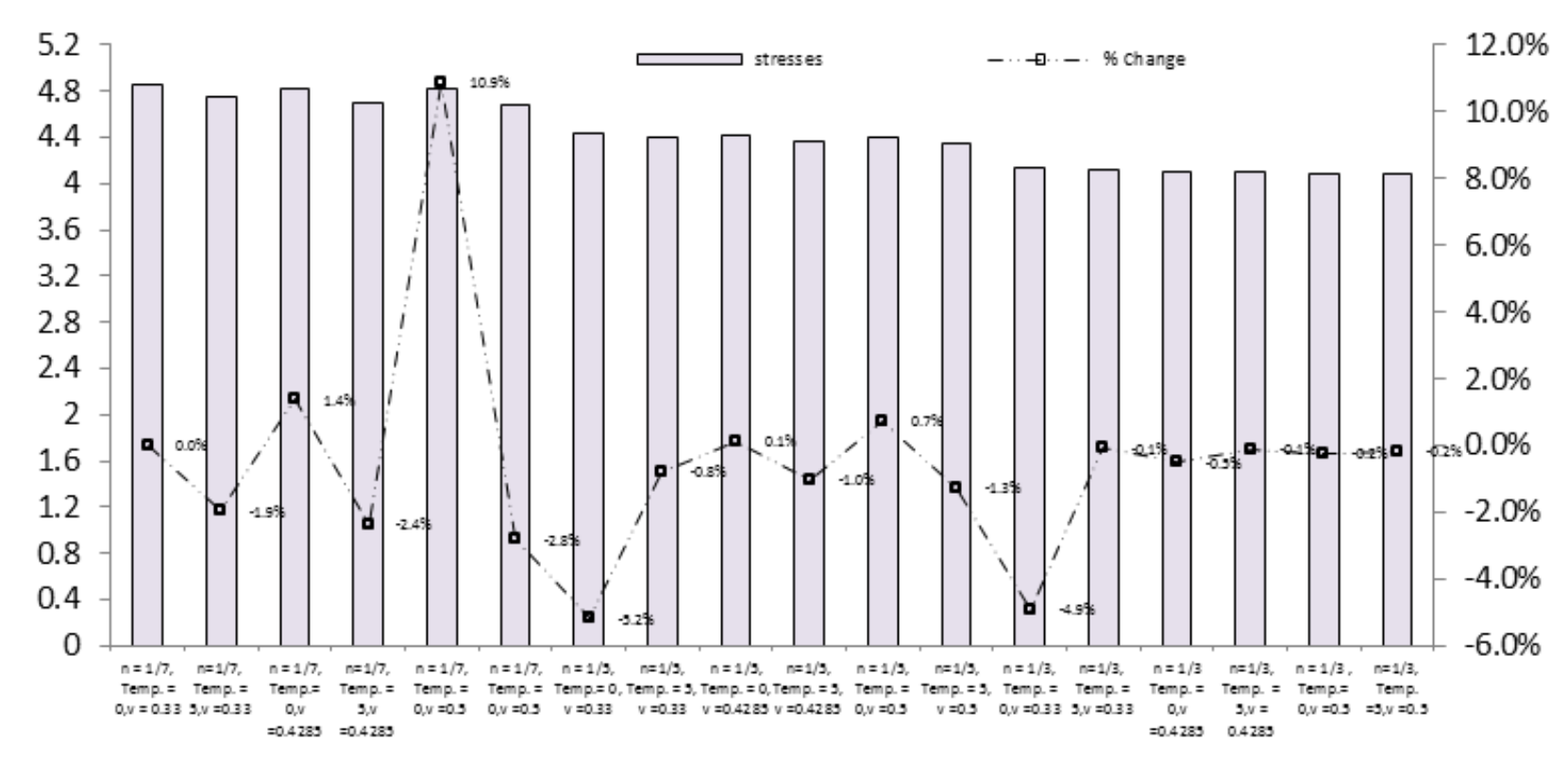

Figure $4(\mathrm{a}) \cdot \Omega^{2}=5$

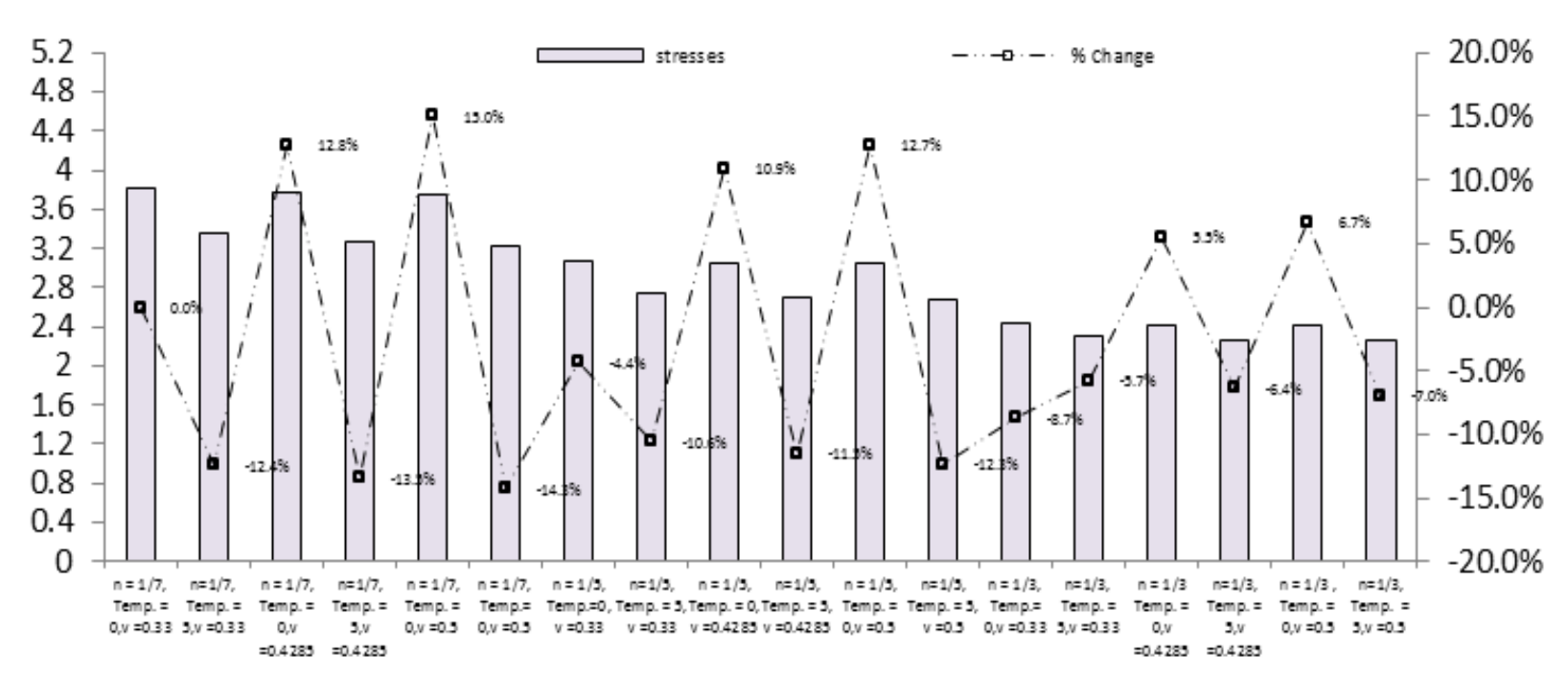

Figure 4(b). $\Omega^{2}=10$

Figures 4(a)-4(b). Percentage change in radial stress with and without temperature at the initial yielding to become fully plastic. 
Table 1. Parentage change stresses for initial yielding and fully plastic state

\begin{tabular}{|c|c|c|c|c|c|c|c|c|c|}
\hline & $\begin{array}{c}\text { Angular } \\
\text { Speed } \\
\Omega^{2}\end{array}$ & $\begin{array}{c}\text { Temperature } \\
\Theta_{1}\end{array}$ & $\begin{array}{c}\text { Measure } \\
n\end{array}$ & \multicolumn{2}{|c|}{$\begin{array}{c}\text { Radial stresses } \\
\sigma_{r}\end{array}$} & \multirow{2}{*}{$\begin{array}{l}\begin{array}{c}\text { Percentage } \\
\text { change } \\
\text { (Decrease) }\end{array} \\
10.6 \%\end{array}$} & \multicolumn{2}{|l|}{$\begin{array}{c}\text { Circumferential } \\
\text { stress } \\
\sigma_{\theta}\end{array}$} & \multirow{2}{*}{$\begin{array}{c}\begin{array}{c}\text { Percentage } \\
\text { change } \\
\text { (Decrease) }\end{array} \\
48 \%\end{array}$} \\
\hline \multirow{18}{*}{ 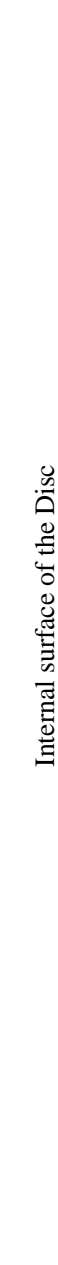 } & \multirow{9}{*}{5} & $\begin{array}{l}0 \\
5\end{array}$ & $\begin{array}{l}1 / 5 \\
1 / 5\end{array}$ & $\begin{array}{l}3.0794932 \\
2.7531297 \\
\end{array}$ & \multirow{3}{*}{$\begin{array}{l}\quad \begin{array}{l}v=0.33 \\
\text { (compressi }\end{array} \\
\text { ble } \\
\text { material) }\end{array}$} & & $\begin{array}{l}-0.679905306 \\
-1.006268796\end{array}$ & \multirow{3}{*}{$\begin{array}{l}\quad v=0.33 \\
\text { (compressi } \\
\text { ble } \\
\text { material) }\end{array}$} & \\
\hline & & $\begin{array}{l}0 \\
5\end{array}$ & $\begin{array}{l}1 / 3 \\
1 / 3\end{array}$ & $\begin{array}{l}2.4367435 \\
2.2967465\end{array}$ & & $5.7 \%$ & $\begin{array}{l}0.180878842 \\
0.0408818\end{array}$ & & $77.4 \%$ \\
\hline & & $\begin{array}{l}0 \\
5\end{array}$ & $\begin{array}{l}1 / 7 \\
1 / 7\end{array}$ & $\begin{array}{l}3.8233112 \\
3.3475945\end{array}$ & & $12.4 \%$ & $\begin{array}{l}-1.471616276 \\
-1.947333004\end{array}$ & & $32.33 \%$ \\
\hline & & $\begin{array}{l}0 \\
5\end{array}$ & $\begin{array}{l}1 / 5 \\
1 / 5\end{array}$ & $\begin{array}{l}3.052207 \\
2.7008862\end{array}$ & $\begin{array}{l}v= \\
0.4285\end{array}$ & $11.5 \%$ & $\begin{array}{l}-0.447968036 \\
-0.799288839\end{array}$ & $\begin{array}{l}v=0.4285 \\
\text { compressi }\end{array}$ & $78.43 \%$ \\
\hline & & $\begin{array}{l}0 \\
5\end{array}$ & $\begin{array}{l}1 / 3 \\
1 / 3 \\
\end{array}$ & $\begin{array}{l}2.4231061 \\
2.2689446 \\
\end{array}$ & $\begin{array}{l}\text { (compressi } \\
\text { ble }\end{array}$ & $6.4 \%$ & $\begin{array}{l}0.322791059 \\
0.168629576 \\
\end{array}$ & $\begin{array}{l}\text { ble } \\
\text { material) }\end{array}$ & $47.76 \%$ \\
\hline & & $\begin{array}{l}0 \\
5\end{array}$ & $\begin{array}{l}1 / 7 \\
1 / 7\end{array}$ & $\begin{array}{l}3.7748112 \\
3.2670218 \\
\end{array}$ & material) & $13.5 \%$ & $\begin{array}{l}-1.155012792 \\
-1.662802181\end{array}$ & & $43.96 \%$ \\
\hline & & $\begin{array}{l}0 \\
5\end{array}$ & $\begin{array}{l}1 / 5 \\
1 / 5\end{array}$ & $\begin{array}{l}3.0448837 \\
2.6699252\end{array}$ & $\begin{array}{c}v=0.5 \\
\text { (Incompres }\end{array}$ & $12.3 \%$ & $\begin{array}{l}-0.288449598 \\
-0.663408086\end{array}$ & $\begin{array}{c}v=0.5 \\
\text { Incompres }\end{array}$ & $129.99 \%$ \\
\hline & & $5^{0}$ & $\begin{array}{l}1 / 3 \\
1 / 3\end{array}$ & $\begin{array}{l}2.420306 \\
2.2518215\end{array}$ & $\begin{array}{l}\text { sible } \\
\text { material) }\end{array}$ & $7 \%$ & $\begin{array}{l}0.420105989 \\
0.251621524\end{array}$ & 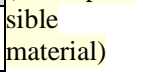 & $40.11 \%$ \\
\hline & & 5 & $\begin{array}{l}1 / 7 \\
1 / 7\end{array}$ & $\begin{array}{l}3.7567948 \\
3.2198703\end{array}$ & & $14.3 \%$ & $\begin{array}{l}-0.938040834 \\
-1.474965389\end{array}$ & & $57.24 \%$ \\
\hline & \multirow{9}{*}{10} & $\begin{array}{l}0 \\
5\end{array}$ & $\begin{array}{l}1 / 5 \\
1 / 5 \\
\end{array}$ & $\begin{array}{l}4.4385543 \\
4.4041066 \\
\end{array}$ & \multirow{3}{*}{$\begin{array}{l}\quad v=0.33 \\
\text { (compressi } \\
\text { ble } \\
\text { material) }\end{array}$} & $0.8 \%$ & $\begin{array}{l}0.67915578 \\
0.644708152 \\
\end{array}$ & \multirow{3}{*}{$\begin{array}{l}\quad v=0.33 \\
\text { (compressi } \\
\text { ble } \\
\text { material) }\end{array}$} & $5.07 \%$ \\
\hline & & $\begin{array}{l}0 \\
5\end{array}$ & $\begin{array}{l}1 / 3 \\
1 / 3\end{array}$ & $\begin{array}{l}4.1302201 \\
4.1270352\end{array}$ & & $0.1 \%$ & $\begin{array}{l}1.874355393 \\
1.8711705\end{array}$ & & $0.17 \%$ \\
\hline & & $\begin{array}{l}0 \\
5\end{array}$ & $\begin{array}{l}1 / 7 \\
1 / 7\end{array}$ & $\begin{array}{l}4.8494276 \\
4.7550385\end{array}$ & & $1.9 \%$ & $\begin{array}{l}-0.445499883 \\
-0.539889002\end{array}$ & & $21.19 \%$ \\
\hline & & $\begin{array}{l}0 \\
5\end{array}$ & $\begin{array}{l}1 / 5 \\
1 / 5\end{array}$ & $\begin{array}{l}4.409522 \\
4.3652161\end{array}$ & \multirow{3}{*}{$\begin{array}{l}v= \\
0.4285 \\
\text { (compressi } \\
\text { ble } \\
\text { material) }\end{array}$} & $1 \%$ & $\begin{array}{l}0.909347033 \\
0.865041045\end{array}$ & \multirow{3}{*}{$\begin{array}{l}v=0.4285 \\
\text { (compressi } \\
\text { ble } \\
\text { material) }\end{array}$} & $4.87 \%$ \\
\hline & & $\begin{array}{l}0 \\
5\end{array}$ & $\begin{array}{l}1 / 3 \\
1 / 3\end{array}$ & $\begin{array}{l}4.1062292 \\
4.1014429\end{array}$ & & $0.12 \%$ & $\begin{array}{l}2.005914208 \\
2.001127907\end{array}$ & & $0.24 \%$ \\
\hline & & $\begin{array}{l}0 \\
5\end{array}$ & $\begin{array}{l}1 / 7 \\
1 / 7\end{array}$ & $\begin{array}{l}.8202831 \\
4.7061754\end{array}$ & & $2.4 \%$ & $\begin{array}{l}-0.109540887 \\
-0.223648537\end{array}$ & & $104.17 \%$ \\
\hline & & $\begin{array}{l}0 \\
5\end{array}$ & $\begin{array}{l}1 / 5 \\
1 / 5\end{array}$ & $\begin{array}{c}4.3979025 \\
4.3425087\end{array}$ & \multirow{3}{*}{\begin{tabular}{l}
\multicolumn{1}{c}{$v=0.5$} \\
(Incompres \\
sible \\
material)
\end{tabular}} & $1.3 \%$ & $\begin{array}{l}1.064569146 \\
1.009175342\end{array}$ & \multirow{3}{*}{\begin{tabular}{l}
\multicolumn{1}{c}{$v=0.5$} \\
Incompres \\
sible \\
material)
\end{tabular}} & $5.2 \%$ \\
\hline & & $\begin{array}{l}0 \\
5\end{array}$ & $\begin{array}{l}1 / 3 \\
1 / 3\end{array}$ & $\begin{array}{c}4.0920804 \\
4.0851875\end{array}$ & & $0.2 \%$ & $\begin{array}{l}2.091880367 \\
2.084987446 \\
\end{array}$ & & $0.33 \%$ \\
\hline & & $\begin{array}{l}0 \\
5\end{array}$ & $\begin{array}{l}1 / 7 \\
1 / 7\end{array}$ & $\begin{array}{l}4.8158733 \\
4.6810347\end{array}$ & & $2.8 \%$ & $\begin{array}{l}0.12103761 \\
-0.01380097\end{array}$ & & $111.4 \%$ \\
\hline
\end{tabular}

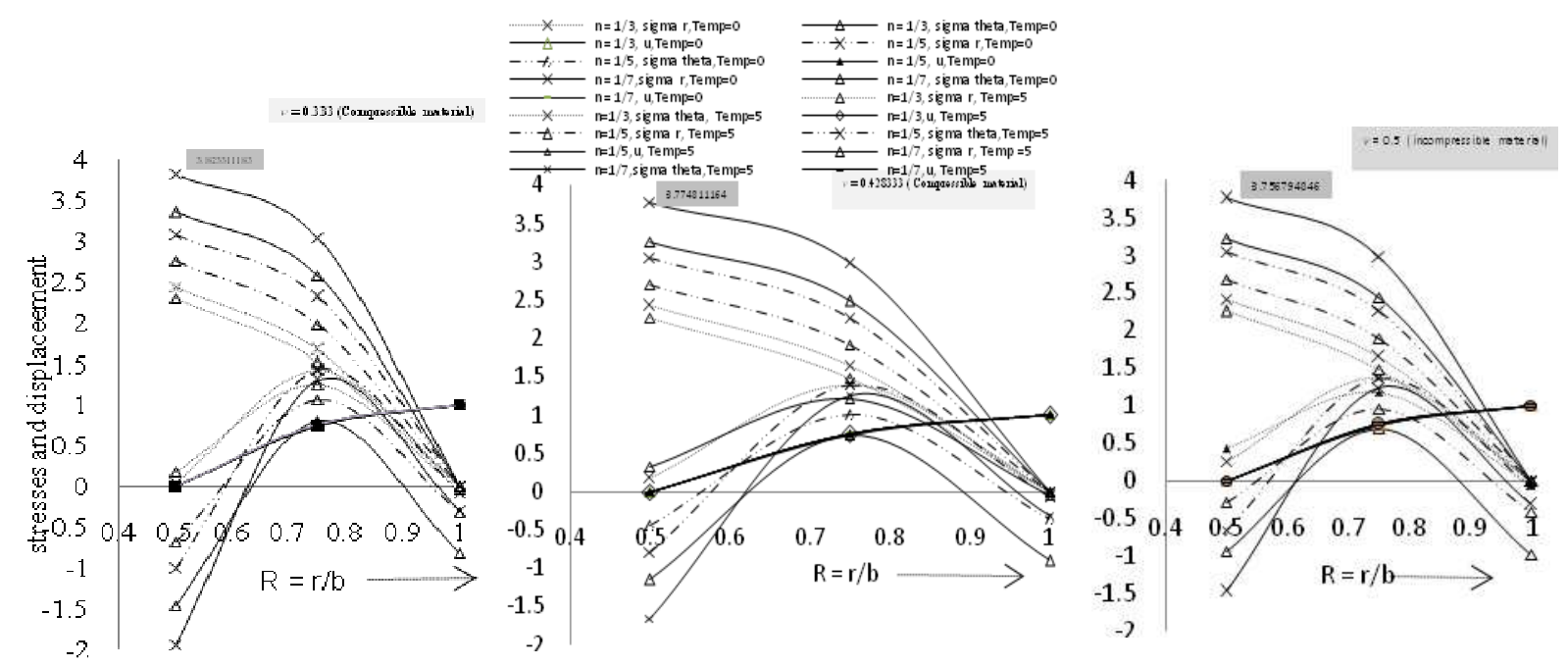

Figure 5(a). Stresses and displacement distribution along the $\mathrm{r}$ adii ratio $\mathrm{R}=\mathrm{r} / \mathrm{b}$ at angular speed $\Omega^{2}=5$. 


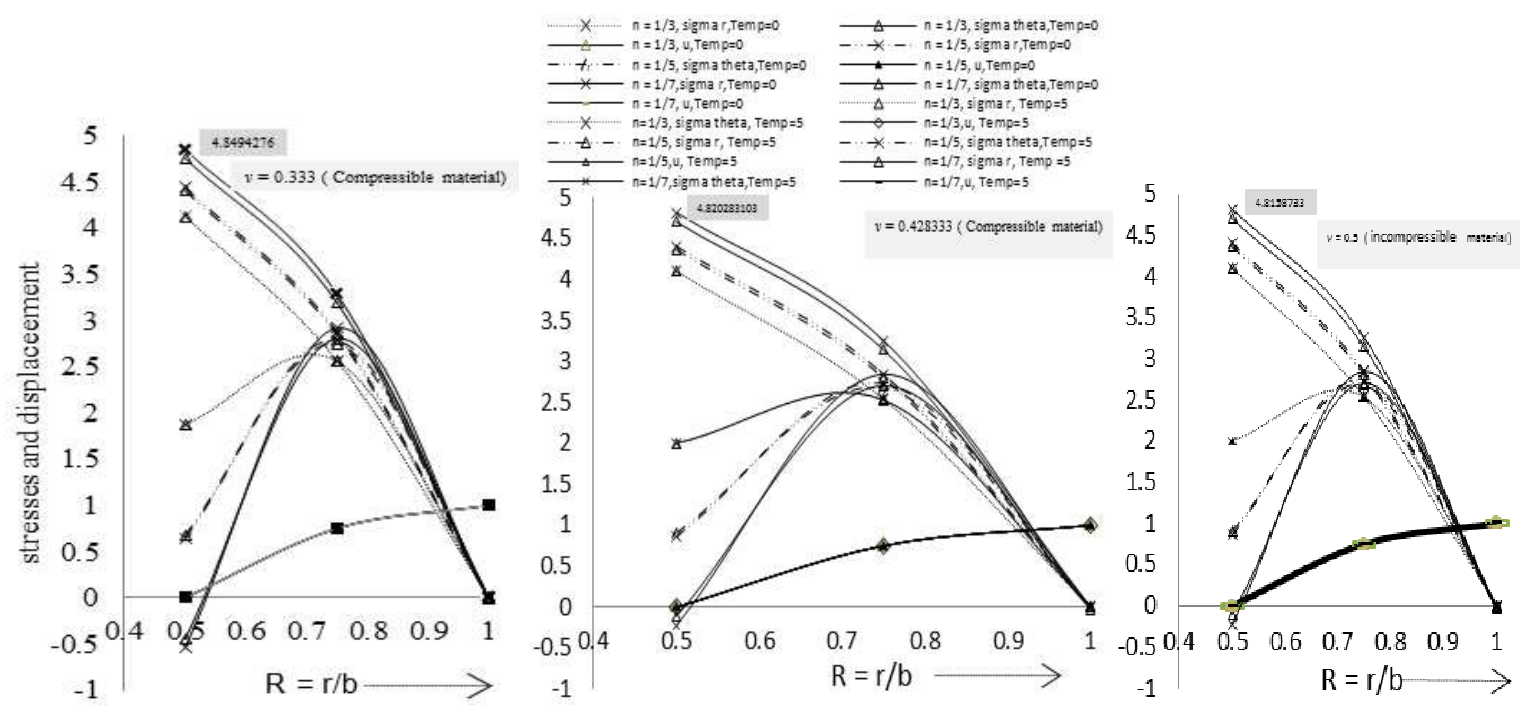

Figure 5(b). Stresses and displacement distribution along the radii ratio $\mathrm{R}=r / b$

$$
\text { at angular speed } \Omega^{2}=10 \text {. }
$$

Curve are produced for strain rates along the radii ratio $\mathrm{R}=\mathrm{r} / \mathrm{b}$ (see Figures $6(\mathrm{a})$ and $6(\mathrm{~b})$ ) for rotating disc made of compressible materials (i.e. saturated clay or copper) as well as incompressible material (i.e. rubber) with angular speed $\Omega^{2}=50$ and 75 for measure $n=1 / 7$, $1 / 5,1 / 3$ ( i.e. $N=7,5,3$ ). It has been seen (Figures 6(a)-6(b)) that rotating disc made of compressible materials has maximum value of strain at the internal surface as compared to disc made of incompressible material for measure $\mathrm{n}=1 / 7,1 / 5,1 / 3$ (i.e. $\mathrm{N}=7,5,3$ ) at angular speed $\Omega^{2}=5$. Since the values of strain rates further increases at the internal surface with increase value of angular speed say $\Omega^{2}=10$ respectively. With the introduction of thermal effects the maximum value of strain rates further increases at the internal surface for compressible materials (i.e. saturated clay or copper) as compare to incompressible material. Measure decrease value of strain rates at the internal surface. Rotating disc is likely to fracture by cleavage close to the shaft at the bore.

\section{CONCLUSION}

Thermal effect decreased value of radial stress at the internal surface of the rotating isotropic disc made of compressible material as well as incompressible material and this value of radial stress further much increases with the increase in angular speed. With the introduction of thermal effects the maximum value of strain rates further increases at the internal surface for compressible materials as compare to incompressible material.

\section{Acknowledgment}

The authors gratefully acknowledge UGC, New Delhi for providing financial support to carry out this research work under UGC-Major Research Project Scheme (MRP-MAJORMATH-2013-41603). 


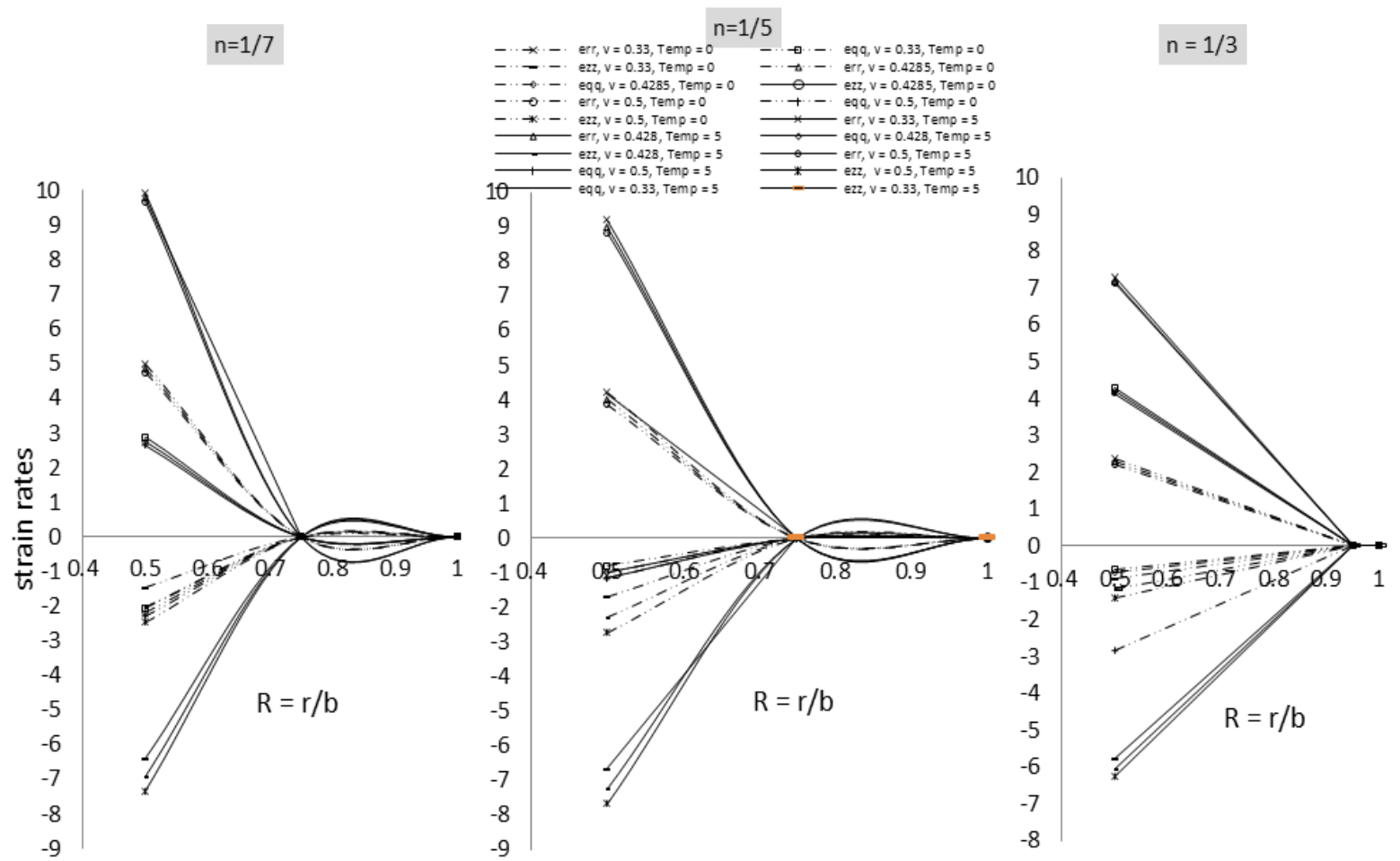

Figure 6(a). Strain rates distribution in disc along the radii ratio $\mathrm{R}=\mathrm{r} / \mathrm{b}$ at angular speed at angular speed $\Omega^{2}=5$.

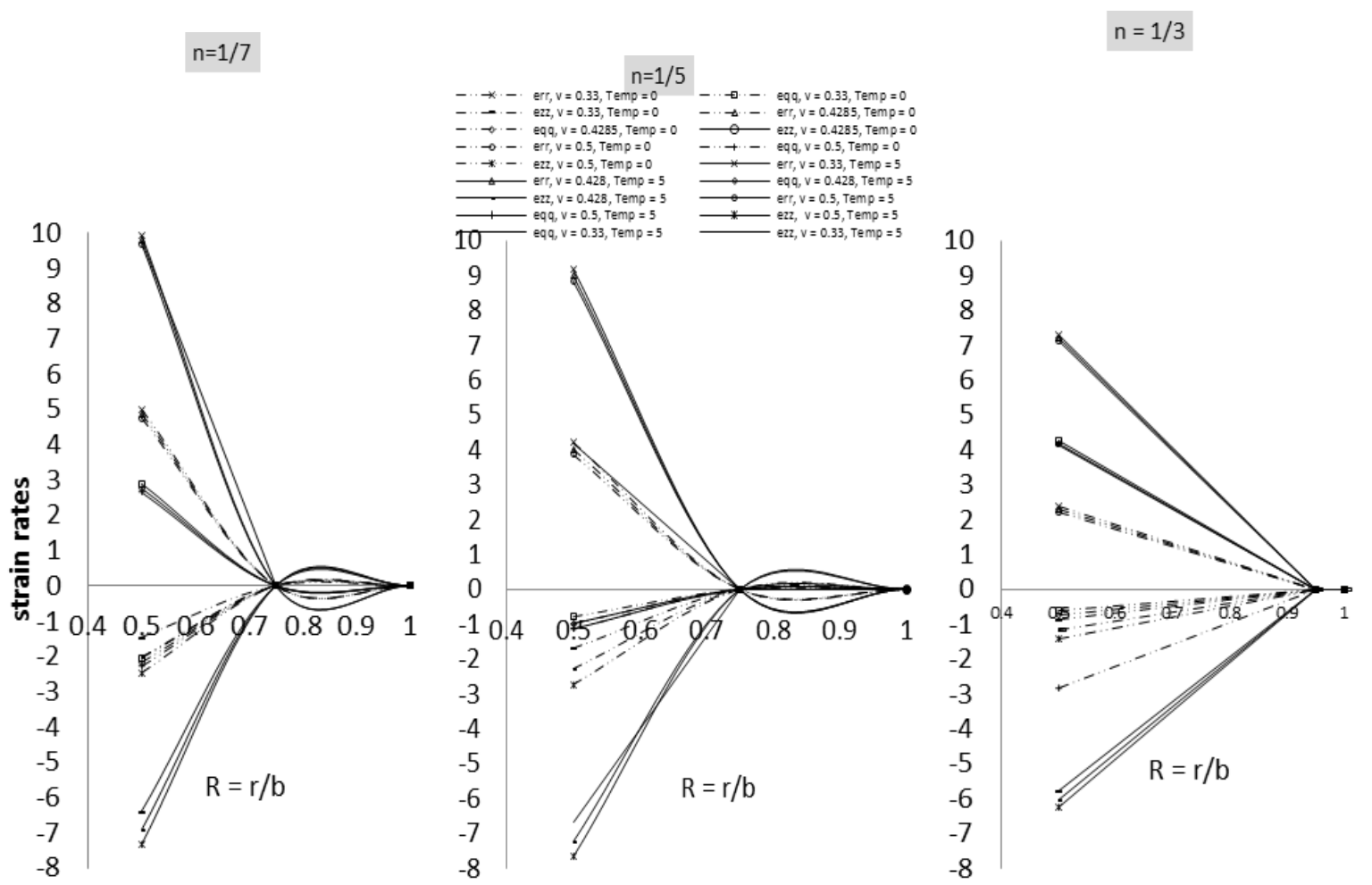

Figure 6(b). Strain rates distribution in disc along the radii ratio $\mathrm{R}=\mathrm{r} / \mathrm{b}$ at angular speed at angular speed $\Omega^{2}=10$. 


\section{References}

[1] Boyle, J.T., Spence, J., Stress analysis for creep, Butterworths, Coy. Ltd. London (1983).

[2] DEEPAK, D., GUPTA, V.K., DHAM, A.K., Creep modeling in functionally graded rotating disc of variable thickness, Journal of Mechanical Science and Technology 24 (1) (2010) 2221-2232.

[3] DeEPaK, D., GarG, M., Gupta, V.K., Creep behavior of rotating FGM disc with linear and hyperbolic thickness profiles, Kragujevac J. Sci. 37 (2015) 35-48.

[4] FARSHI, B., JAHED, H. and MEHRABIAN, A., Optimum design of inhomogeneous nonuniform rotating discs, Computers \& Structures 82 (9-10) (2004) 773-779.

[5] FARSHI, B., BIDABADI, J., Optimum design of inhomogeneous rotating discs under secondary creep, International Journal of Pressure Vessels and Piping 85 (7) .(2008) 507-515.

[6] Gupta, V.K., Kwatra, N., Ray, S., Artificial neural network modeling of creep behavior in a rotating composite disc, Engineering Computations 24 (2) (2007) 151164.

[7] Gupta, S.K., Dharmani, R.L., Creep transition in thick walled cylinder under internal Pressure, Zeitschrift für Angewandte Mathematik und Mechanik 59 (10) (1979) 517521.

[8] Gupta, S.K., Dharmani, R.L., Creep Transition in Rotating Cylinders, Indian J. Pure Applied Math. 15 (6) (1981) 525-536.

[9] Gupta, S.K. PathaK Sonia, Creep transition in a thin rotating disc of variable density, Defence Sci. Journal., India 50 (2) (2000) 147-153.

[10] Gupta S.K., Sharma Sanjeev, Pathak Sonia, Creep transition in thin rotating disc having variable thickness and variable density, Indian J. Pure and Appl. Math. 31 (10) (2000) 1235-1248.

[11] GuPTA, S.K., ThakUR PANKaJ, Creep transition in a thin rotating disc with rigid Inclusion, Defence Science Journal, India, 57 (2) (2007) 185-195.

[12] GuPTA S.K., ThakUR PANKaJ, Creep transition in an isotropic disc having variable thickness subjected to internal pressure, Proceeding National Academy of Science, India, Section-A 78 (Part-1) (2008) 57-66.

[13] Hoffman,O., SACHS G., Introduction to theory of plasticity for engineers, Literary Licensing, LLC (2012) 99-104.

[14] Kraus, H., Creep Analysis, Wiley Publication, New York, USA, (1980) pp. 195-198.

[15] Laskaj, M., MurPhy, B., Houngan, K., Improving the efficiency of cooling the front disc brake on a V8 racing car, "Project report", Monash University, Melbourne (1999).

[16] Levitsky, M., ShafFer, B.W., Residual thermal stresses in a solid sphere from a thermosetting material, Jr. of Appl. Mech., Trans. of ASME 42 (3) (1975) 651-655.

[17] Lubhan, D., Felger, R.P., Plasticity and creep of Metals, Wiley, New York, USA (1961) 568-599.

[18] NABARro, F.R.N., Villiers H.L., De Physics of Creep, Taylor \& Francis, PA (1995). 
[19] OdQuist, F.K.G., Mathematical theory of creep and creep rupture, Clarendo Press, Oxford, USA (1974).

[20] PARKUS, H., Thermo-Elasticity, Springer - Verlag, Wien, New York, USA (1976).

[21] Penny, R.K., MariotT, D.L., Design for Creep, Chapman and Hall. London (1995).

[22] SETH, B.R., Transition theory of elastic-plastic deformation, creep and relaxation, Nature, 195 (1962) 896 -897, DOI:10.1038/195896a0.

[23] SETH, B.R., Measure concept in Mechanics, International Journal of Non-linear Mechanics 1 (1) (1966) 35-40.

[24] SETH, B.R., Creep rupture, IUTAM Symposium on Creep in structures, Gothenburg, Sweden (1970) 167-169.

[25] SeTH, B.R., Creep transition, J. Math. Phys. Sci. 6 (2) (1972) 1-5.

[26] SETH, B.R., Creep transition in rotating cylinder, J. Math. Phys. Sci. 8 (1) (1974) 2-6.

[27] Sharma SANJEeV, SAHNi MANOJ, Creep deformation of a thin rotating disk of exponentially varying thickness with inclusion, Proceedings of the $20103 \mathrm{rd}$ International Conference on Emerging Trends in Engineering and Technology, IEEE Computer Society Washington, DC, USA, (2010) 271-276, DOI: 10.1109/ICETET.2010.52.

[28] Sharma Sanjeev, Sahai Ila, Kumar Ravindra, Creep transition of a thin rotating annular disk of exponentially variable thickness with inclusion and edge load, Procedia Engineering 55 (2013) 348-354.

[29] ShKulA, R.K., Creep transition in a thin rotating non-homogeneous disc, Indian J. pure and appl. Math. 27 (5) (1996) 487-498.

[30] ThaKUR PANKAJ, Creep transition stresses in a thin rotating disc with shaft by finite deformation under steady state temperature, Thermal Science 14 (2) (2010) 425-436.

[31] Thakur PankaJ, Singh, S.B., SaWhney S., Elastic-plastic infinitesimal deformation in a solid disk under heat effect by using Seth theory, Int. J. Appl. Comput. Math. (2015) DOI: 10.1007/s40819-015-0116-9.

[32] Thakur Pankaj, Singh, S.B., Kaur, J., Thermal Creep stresses and strain rates in a circular Disc with shaft having variable density, Engineering Computation 33 (3) (2016) 698-712.

[33] WaHL, A.M., Analysis of creep in rotating discs based on Tresca criterion and associated flow rule, Jr. Appl. Mech. 23 (1956) 103-106. 